This item was submitted to Loughborough's Research Repository by the author.

Items in Figshare are protected by copyright, with all rights reserved, unless otherwise indicated.

\title{
Atmospheric plasma inactivation of biofilm-forming bacteria for food safety control
}

PLEASE CITE THE PUBLISHED VERSION

PUBLISHER

(C) IEEE

LICENCE

CC BY-NC-ND 4.0

\section{REPOSITORY RECORD}

Vleugels, Mieke, Gilbert Shama, Xu-Tao Deng, Elizabeth Greenacre, Tim Brocklehurst, and Michael G. Kong. 2008. "Atmospheric Plasma Inactivation of Biofilm-forming Bacteria for Food Safety Control". figshare. https://hdl.handle.net/2134/3481. 
This item was submitted to Loughborough's Institutional Repository by the author and is made available under the following Creative Commons Licence conditions.

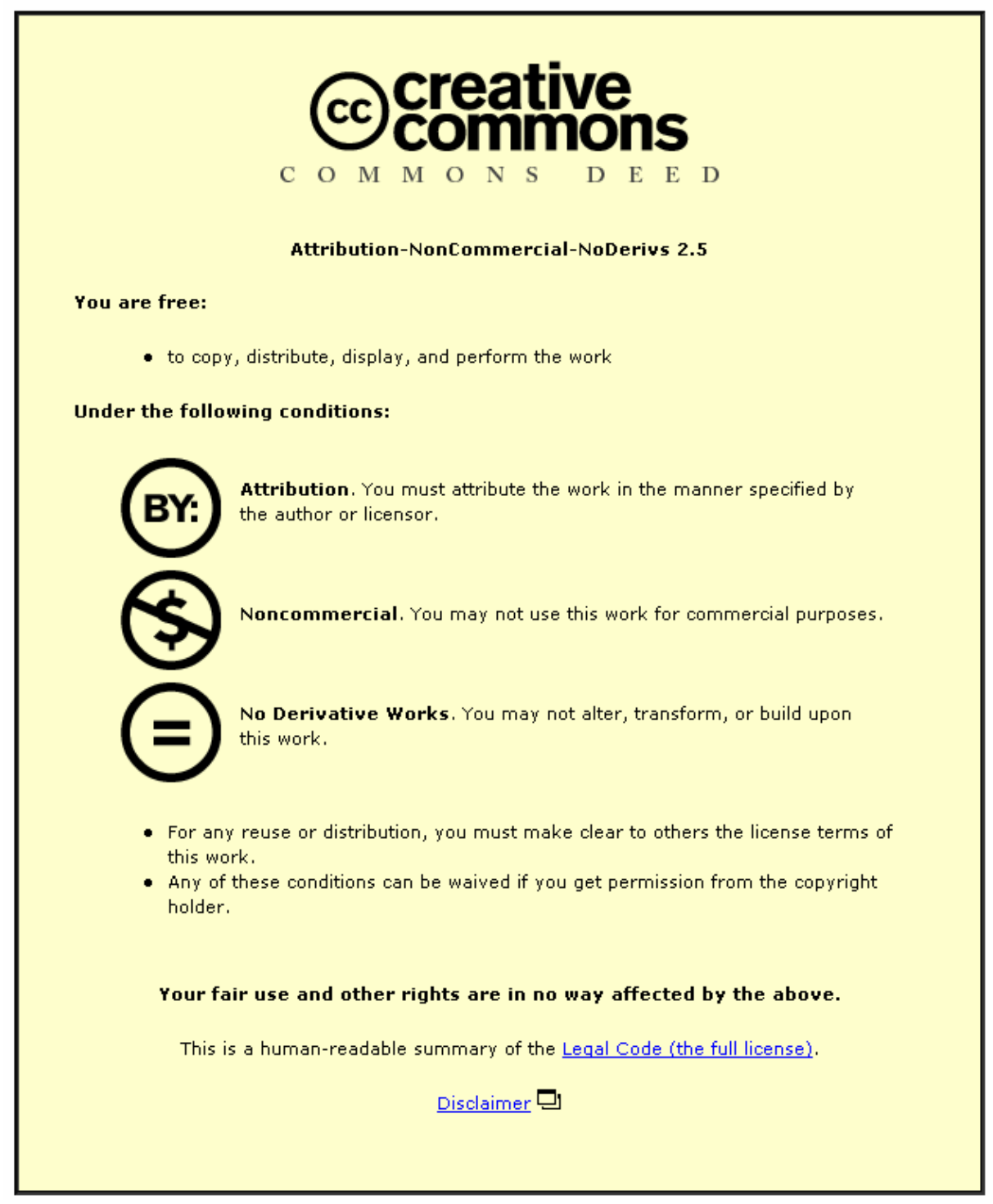

For the full text of this licence, please go to: http://creativecommons.org/licenses/by-nc-nd/2.5/ 


\title{
Atmospheric Plasma Inactivation of Biofilm-Forming Bacteria for Food Safety Control
}

\author{
Mieke Vleugels, Gilbert Shama, Xuetao T. Deng, Elizabeth Greenacre, Tim Brocklehurst, and Michael G. Kong
}

\begin{abstract}
The ability of atmospheric pressure glow discharges (APGD) to inactivate microorganisms has been demonstrated in a number of previous studies. However, most of this work has been performed using microorganisms that do not form biofilms and with the microorganisms supported on abiotic surfaces that discourage cell growth. When microorganisms attach to the surface of a food, they can extract nutrients from the food and proliferate at the surface. Often this growth takes the form of biofilms which comprise three-dimensional (3-D) networks of polysaccharides that attach microorganisms to surfaces and serve to protect them from external stresses; fresh foods, such as salad crops, frequently harbor biofilms. We believe that the use of APGD offers a potential for inactivating microorganisms on the surface of fresh foods that cannot be readily treated by other methods without inducing unacceptable changes to these foods. As a first step toward a full evaluation of the viability of the APGD technology for food safety control, we consider in this paper two key issues, namely: 1) whether atmospheric glow discharges can inactivate biofilm-forming microorganisms and 2) whether plasma treatment causes significant discoloration to food surfaces. Using the biofilm-forming bacterium Pantoea agglomerans and bell peppers (Capsicum annuum) as a typical example of plant tissue, we show that atmospheric $\mathrm{He}-\mathrm{O}_{2}$ plasmas can be effective inactivation agents without causing unacceptable levels of discoloration to the peppers, and that furthermore they are superior to the use of low-pressure ultraviolet sources.
\end{abstract}

Index Terms-Atmospheric glow discharges, biofilm, decontamination, food-borne microorganisms, food surfaces, inactivation, sterilization.

\section{INTRODUCTION}

A TMOSPHERIC pressure glow discharges (APGD) have been shown to be capable of inactivating microorganisms and surface sterilization [1]-[11]. Different from low-pressure plasmas generated in a vacuum chamber, key inactivation agents in atmospheric glow discharges are thought to be largely reactive chemical species, such as atomic oxygen, ozone, hydroxyl radicals, as well as charged particles. By comparison, ultraviolet (UV) photons play a less important role in APGD because they are easily absorbed by gas atoms and molecules at atmospheric pressure. While microorganisms successfully inactivated by atmospheric plasmas include both gram-negative and gram-positive bacteria, most APGD sterilization studies reported so far

Manuscript received September 14, 2004; revised November 23, 2004

M. Vleugels and G. Shama are with the Department of Chemical Engineering, Loughborough University, Leics LE11 3TU, U.K.

X. T. Deng and M. G. Kong are with the Department of Electronic and Electrical Engineering, Loughborough University, Leics LE11 3TU, U.K. (e-mail: m.g.kong@lboro.ac.uk).

E. Greenacre and T. Brocklehurst are with the Institute of Food Research, Norwich NR4 7UA, U.K.

Digital Object Identifier 10.1109/TPS.2005.844524 have been carried out on abiotic surfaces that discourage cell growth. In practice, microorganisms are often embedded in the nutrient-rich environment of biofilms where they are able to proliferate and are largely protected from external stresses such as direct attack of plasma species. Therefore, it is important to study the capability of APGD to inactivate microorganisms embedded in biofilms.

This paper addresses the above issue in the context of foodborne microorganisms with an ultimate objective of establishing whether APGD can decontaminate fresh foods without significantly affecting the nutrient content and textural qualities of the foods. Given that the biological lethality of APGD depends on the flux of their reactive species and charged particles, a high flux of plasma species is desirable. Yet, simultaneously, a high concentration of reactive plasma species poses a large threat to the material integrity of the surface that hosts the microorganisms. Also, the production of such species is often associated with high gas temperature, which can cause unacceptable thermal damage to fresh foods. Therefore, the goal of achieving high inactivation efficacy cannot be pursued in isolation and any plasma conditions that lead to improved inactivation efficacy must be evaluated against their implication for surface damage. In Section II, we consider the contrasting need for inactivation efficacy and damage mitigation to deduce key constraints in the application of APGD to fresh food decontamination. Based on this, justifications are presented for the choice of our APGD system, representative food-borne microorganism, and two aspects of surface damage. Then, in Section III, we report the results of our inactivation tests using both a room-temperature APGD and a UV lamp. Using bell peppers to represent a typical fresh food surface, we report in Section IV on tests conducted to assess the dehydration and discoloration effects on the peppers by both plasma treatment and by the flow of unionized gases. Finally, in Section V, the findings discussed in the previous sections are summarized.

\section{CONSTRAINTS FOR FOOD DECONTAMINATION}

Cut and prepackaged fresh vegetable and fruit salad products have traditionally been decontaminated using chlorine. Its decontamination capability may be illustrated by a study in 1999 , in which a $\log$ reduction of 1.75 in the microflora present on shredded iceberg lettuce was achieved after an exposure time of $7 \mathrm{~min}$ in chlorinated water at $43^{\circ} \mathrm{C}$ [12]. Reviewing work done on the elimination of $E$. coli $\mathrm{O} 157$ from the surface of whole apples, Du et al. observed that aqueous sanitizers such as chlorine, hydrogen peroxide, trisodium phosphate, peroxyacetic acid, and acetic acid used alone or in combination did not significantly reduce the surface counts of this pathogen [13]. 


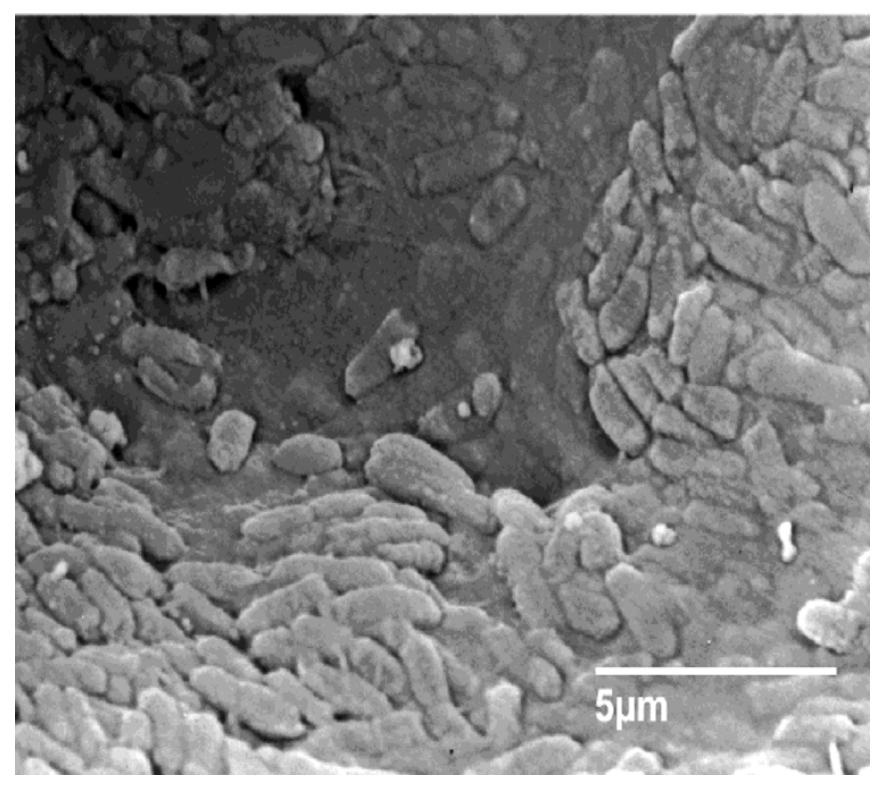

Fig. 1. SEM image of a microbial biofilm on the cut surface of green bell pepper.

Although chlorine is of only limited efficacy as a microbiocide and is associated with health risks to humans, no satisfactory alternative to it has yet emerged [14]. In food decontamination, one important complication is the interaction between microbial cells and the surface. This interaction can take many different forms: microorganisms may adsorb to the surface of foods and simply remain attached to it during processing as is the case with abiotic materials. Alternatively, adherent organisms may exploit nutrients present on the surface of foods and go on to proliferate at the surface. Others may, under certain conditions, exude polysaccharides which serve to protect them from environmental stresses and, incidentally, makes it more difficult to detach them from surfaces. This three-dimensional (3-D) matrix of polysaccharides and microorganisms is referred to as a "biofilm." Fig. 1 shows a scanning electron microscope (SEM) image of microorganisms aggregated in a polysaccharide biofilm layer. Apart from stimulating microorganism growth, the biofilm prevents plasma species from direct access to microorganisms that are embedded within the biofilm structure. Therefore, biofilm-forming bacteria present an additional resistance to any decontamination technique. This requires plasma species generated in APGD to have a particularly high level of flux density and biological lethality. In other words, high concentrations of oxygen species, such as atomic oxygen and ozone, are preferred, thus favoring atmospheric plasmas generated in oxygen-rich gases including air rather than in noble gases. It is worth noting that microorganisms in Fig. 1 do not have a uniform surface density and, as such, may present a spatial variation in their protection by the biofilm against attacks of incoming plasma species.

Effective food decontamination will not be acceptable to consumers if it is achieved at the expense of food quality. Quality loss can include loss in nutrient content, and changes in appearance or to textural qualities. It is known that gas temperatures of APGD generated in oxygen-rich gases tends to be higher than that in noble gases. Many fresh foods, particularly fresh salads,

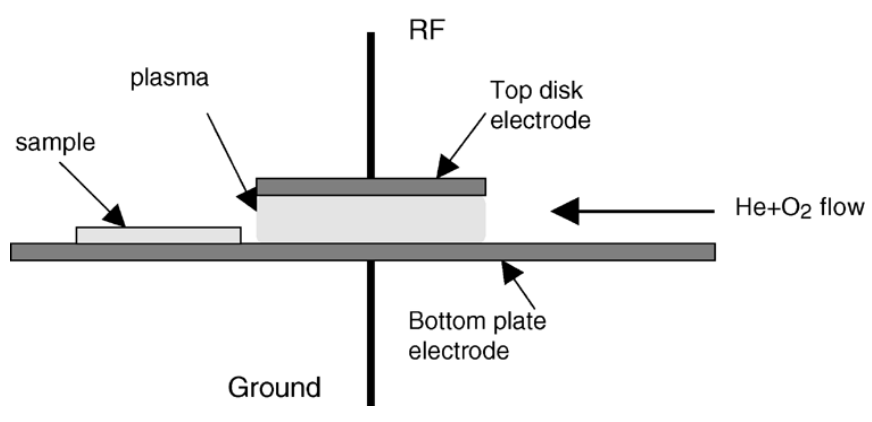

Fig. 2. Schematic of the plasma decontamination rig.

have a very low threshold for permanent thermal damage, and as a general rule inactivation needs to be near room temperature. Consequently, a simultaneous balance is essential between maximization of inactivation efficacy and minimization of food damage. To this end, we used helium-oxygen mixture as the discharge medium with helium used to control thermal runaway of the plasma and oxygen to provide the necessary oxygen species. To reduce gas temperature further, the gas mixture was made to flow through the discharge region. Furthermore, we employed a parallel-plate electrode configuration excited at $460 \mathrm{kHz}$.

To evaluate the inactivation efficacy of $\mathrm{He}_{2} \mathrm{O}_{2}$ APGD, our model system comprises the biofilm-forming bacterium Pantoea agglomerans growing on synthetic membranes to simulate bacterial growth on the surface of plant tissue. $P$. $a g$ glomerans is commonly associated with plant tissues, and is a motile, gram-negative, rod-shaped microorganism [15]. As the biofilm develops with time, the production of polysaccharides becomes more extensive and cells contained within the matrix are shielded to an increasing degree. For our experiments, we consider two cases, namely, P. agglomerans biofilms of $12 \mathrm{~h}$ old and biofilms of $24 \mathrm{~h}$ old. For evaluation of the extent of possible surface damage, this study only considers dehydration and discoloration as an initial indicator and our food model system is a single species of plant tissue (bell peppers).

\section{P. AGGLOMERANS INACTIVATION}

Our atmospheric APGD apparatus has a top disk electrode of 3-cm diameter and a bottom rectangular plate electrode of $5 \times 10 \mathrm{~cm}^{2}$ separated by a gap of typically $0.5 \mathrm{~cm}$, as shown in Fig. 2. The electrode unit is housed within a Perspex box to minimize environmental influence. Plasma excitation is fixed at $460 \mathrm{kHz}$. A helium-oxygen mixture is fed to flow through the gap between the two electrodes with the helium flow rate typically at $5 \mathrm{slm}$ and the oxygen flow rate typically at $5 \mathrm{sccm}$. Dissipated power density is typically $0.5 \mathrm{~W} / \mathrm{cm}^{3}$. For a given helium flow rate, we have measured the temperature of the gas effluent, using a thermocouple, from the plasma generation region at different oxygen flow rates. It has been found necessary to keep the oxygen flow rate at no more than $5 \mathrm{sccm}$ in our system as above this the gas temperature exceeds $70^{\circ} \mathrm{C}$ after some $30 \mathrm{~min}$ and the plasma becomes unstable. As a result, our experiments are carried out for a fixed helium flow of $5 \mathrm{slm}$ and a fixed oxygen flow rate of $5 \mathrm{sccm}$. As a further measure against undesirable thermal impact, we placed the samples in the immediate downstream area of the plasma region and, 


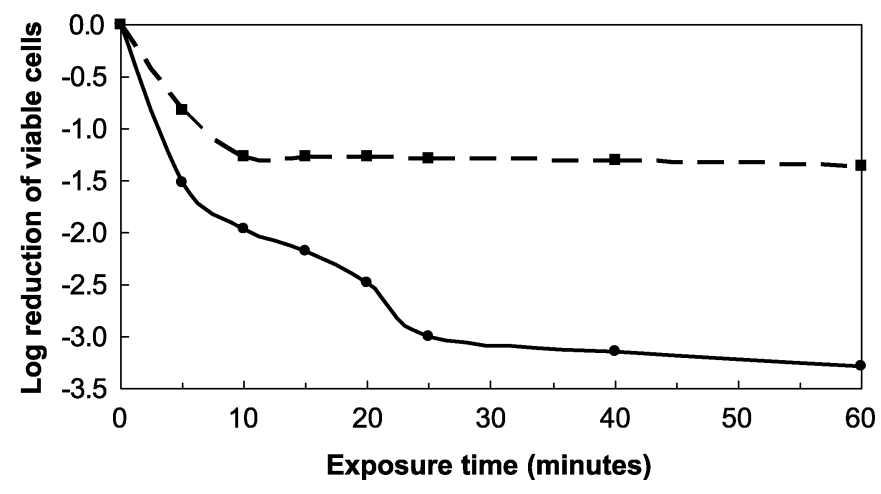

Fig. 3. Inactivation kinetics of a $\mathrm{He}-\mathrm{O}_{2}$ APGD for 12-h-old Pantoea agglomerans samples (solid curve) and 24-h-old Pantoea agglomerans samples (dashed curve).

therefore, our plasma treatment is of a remote nature. $P$. agglomerans was first cultured on circular synthetic membrane having a diameter of $1 \mathrm{~cm}$. The center of the membrane was placed $2 \mathrm{~cm}$ away from the center of the generated APGD. Inactivation efficacy was calculated by measuring the viable cells before and after plasma treatment using standard spread plate methods. All measurements were performed in triplicate. Control tests were performed with the $\mathrm{He}-\mathrm{O}_{2}$ gas flow at $5 \mathrm{slm}$ but with the plasma-generating power supply switched off. Results of control tests showed only very small reductions in the number of viable cells, probably due to the most loosely attached cells being blown off the biofilm surface.

Fig. 3 shows $\log$ reduction of viable cells for $P$. agglomerans in two different biofilms: a 12-h sample set and a 24-h sample set. Each treatment condition was repeated three times with standard deviations typically at $7 \%$ of the mean value. For the 12-h sample set, the inactivation kinetics are seen to have three distinctively different phases: an initial rapid reduction phase, then a slower reduction phase, and finally, a nearconstant plateau. For the 24-h samples, there are only two obvious phases. These kinetic phases suggest different inactivation mechanisms, each of which may be triggered by different plasma species or their different combinational/synergistic effects. While quantitative information on densities and fluxes of plasma species is needed to establish their correlation to the inactivation kinetics of Fig. 3, it is conceivable that the first phase is likely to be related to the inactivation of naked $P$. agglomerans bacteria on the biofilm surface, or the first layer, that can be directly accessed by plasma species. In this first phase, the slower inactivation rate for the 24-h samples is due to the fact that they are thicker biofilms and so their naked $P$. agglomerans bacteria form proportionally less of the total number of cells than that in the 12-h samples. As a result, proportionally fewer bacteria are inactivated for the 24-h samples.

In the second phase for the 12-h samples, it is possible that plasma species can access some bacteria that lie immediately underneath the surface layer of the biofilm but are not well protected. The weak protection to this second layer of bacteria may be a result of an unevenly distributed top layer, as indicated in Fig. 1, and a result of the removal of some plasma-killed cells on the surface layer by the gas flow, both exposing sites of the second layer to plasma species. As not all bacteria on the

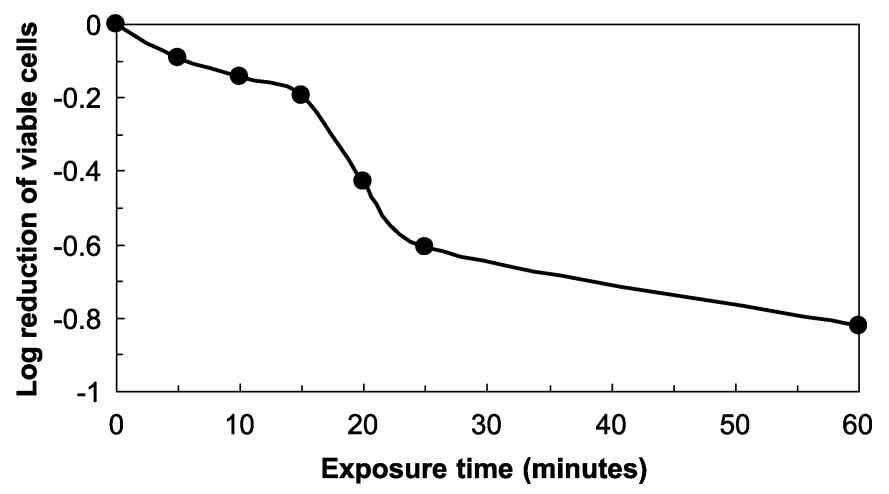

Fig. 4. UV inactivation kinetics of 12-h-old Pantoea agglomerans samples.

second layer immediately beneath the surface layer are exposed to plasma species, the inactivation rate in this phase is lower than that in the first phase. After inactivation of these less-protected bacteria is completed, the inactivation rate is slowed down further to nearly zero. The absence of the second phase for the 24-h samples may be due to a denser surface density of microorganisms achieved with a longer cell growth period that exposes to plasma species very few bacteria on the second layer of the biofilm. As a measure of inactivation efficacy, plasma treatment of 12-h samples is seen in Fig. 3 to induce two orders of log reduction in $10 \mathrm{~min}$. Possible inactivation mechanisms are likely to be associated to reactive species, such as atomic oxygen and $\mathrm{OH}$ radicals, produced by the plasma since UV photons get absorbed easily in atmospheric air and charged particles cannot easily access the sample in its downstream position in Fig. 2.

As a comparison study, we have conducted inactivation tests using a UV lamp (Philips TUV $4 \mathrm{~W}-\mathrm{E}$ m3D). This was a lowpressure mercury vapor lamp emitting principally at $253.7 \mathrm{~nm}$, and at a power density of $0.65 \mathrm{~mW} / \mathrm{cm}^{2}$. Again, for each treatment condition, three samples are used to give a mean value with standard deviation typically at $9 \%$ of the mean data. Fig. 4 shows results of UV inactivation for the 12-h samples only since UV treatment is found ineffective for the 24-h samples. It is clear that a persistent UV irradiation of 60 min result in only 0.8 order of log reduction, much less effective than the plasma treatment. Interestingly, there are also three inactivation phases in Fig. 4. While their associated mechanisms are scientifically interesting, the low inactivation capability of the UV lamp makes it practically irrelevant.

\section{Surface Color Measurements}

Plasma inactivation by two orders of log reduction shown is a sufficiently interesting baseline point for food safety control, for which decontamination requirements are less stringent than sterilization of medical devices. Although we believe that we can improve on this level of inactivation by experimenting still further with different gas compositions, it is important to assess what effects the current treatment has on the surface color of our food samples. For a full evaluation, additional issues concerning food quality must be considered and these include changes in nutrient content and textural qualities, toxic residues, and other 


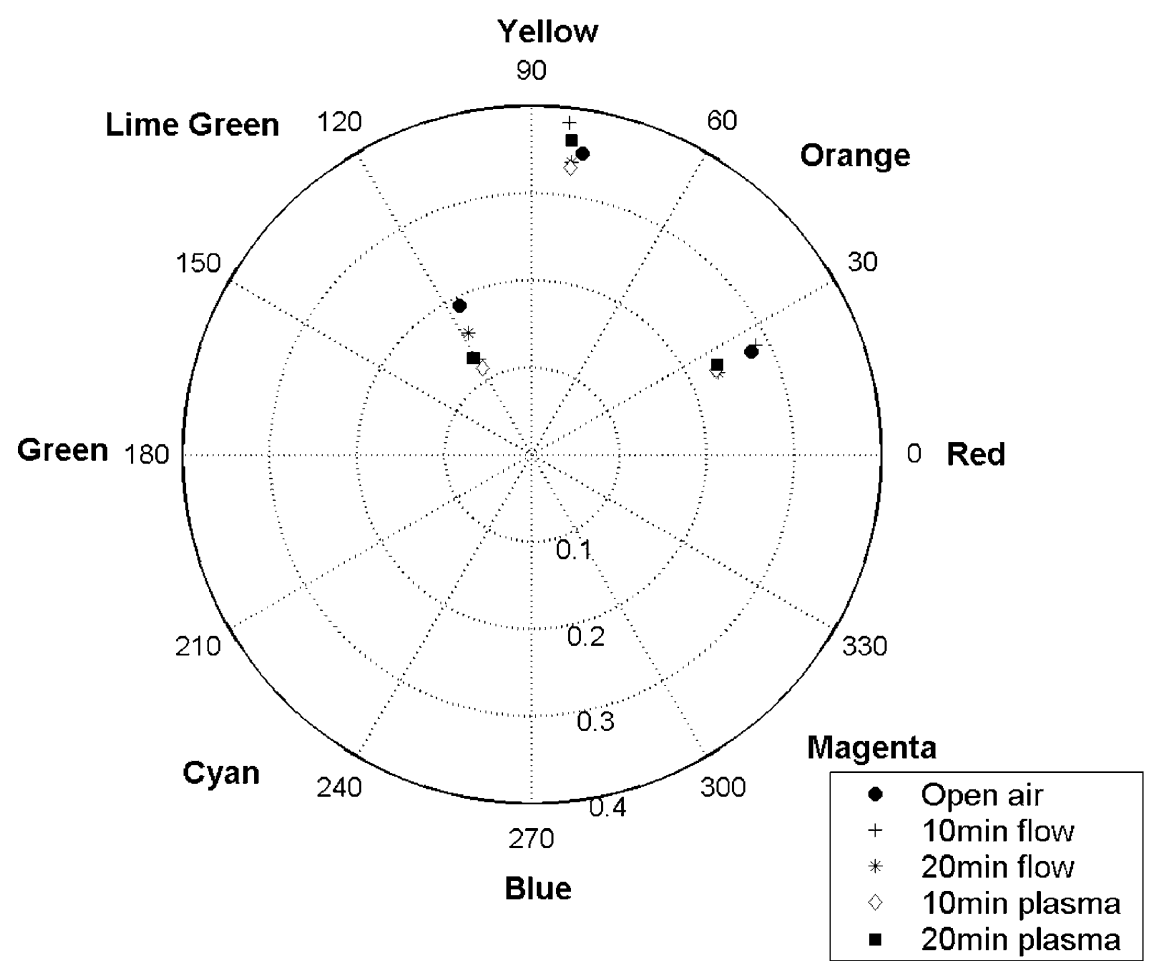

Fig. 5. Chroma-hue plot of pepper samples left in the open air (filled circles), samples in unionized gas flow for 10 min (crosses) and 20 min (stars), and samples in ionized gas flow for $10 \mathrm{~min}$ (diamonds) and $20 \mathrm{~min}$ (squares).

chemical changes. An exhaustive investigation of all these issues is beyond the scope of this paper, and we have confined ourselves here to assessing the effects on surface color of the treatments used to achieve inactivation. If plasma treatment causes an unacceptable level of dehydration or discoloration, it is unlikely to become an acceptable technology for the food industry, regardless of its performance on other food quality indicators.

We used bell peppers here because they represent a commonly used component of precut, ready-to-eat salad mixtures of the type that we believe APGD technology may be directed toward and because they occur in different colors and can highlight changes to color across a significant part of the visible spectrum. Samples of 1-cm diameter were cut from whole fresh peppers using a cork borer. Three sets of samples were taken from red, green, and yellow peppers. We considered three treatment regimes, namely: 1) pepper samples left in ambient air for $20 \mathrm{~min}$; 2) pepper samples treated with unionized gas flow without plasma; and 3) pepper samples treated with ionized gas flow (with plasma). The control samples constituted those from the first treatment regime with samples left in air, and for each treatment condition replicates of ten samples were used. In all the cases considered here, dehydration was found to have had no effect on the peppers as determined by visual inspection and so no further quantification was sought.

To measure discoloration quantitatively, we used a reflectance spectrophotometer (ColorQuest XE, HunterLab). Fig. 5 shows the chroma-hue plot, in which the angle is used to indicate color and the distance from the origin is used to measure color saturation (i.e., the color intensity). Therefore, a variation in the angle among different treatments suggests a color change and a variation in the distance of treatment data from the origin indicates a shift in the shade of the nominal color. Clearly, it is desirable for these changes to be as small as possible. Three sets of data are presented, corresponding to red, yellow, and green peppers with their hue angle at $26^{\circ}, 80^{\circ}$ and $116^{\circ}$, respectively, in Fig. 5. For each of the three data sets aligned along these three hue angles, there are five data each representing the average of ten pepper samples. In the case of red peppers, the plasma-treated samples have very similar color saturation to that of samples treated with unionized gas flow for $20 \mathrm{~min}$. Also, all data appear to align along the same hue angle, suggesting little discoloration. For green peppers, the plasma treated samples have very similar color saturation to that of an ionized gas flow for $10 \mathrm{~min}$. There is, however, a small but clear change in the hue angle. For yellow peppers, the difference in color saturation of plasma treated samples from that of control is smaller than the difference between control and unionized gas treated samples. Therefore, there is little discoloration due to plasma treatment. The change in the hue angle for yellow peppers was smaller than that seen for green peppers.

The results of the chroma-hue plots suggest that the green pepper samples have the largest color variation among the three sample sets. We measured the optical reflectance from the green pepper samples at different wavelengths in the visible range, again using the ColorQuest XE spectrophotometer. As shown in Fig. 6, plasma treatments do reduce the intensity of the reflected optical signal over the visible spectrum from 400 to 700 $\mathrm{nm}$ and the intensity reduction is very similar to that of the case of a 10-min unionized gas flow. To understand the significance of these color variations, we observed that bell peppers in supermarkets differ naturally from one another in terms of color, possibly a result of difference in ripeness and growing conditions. Therefore, we took ten control samples of green peppers and represented their color variation in the form of a variation 


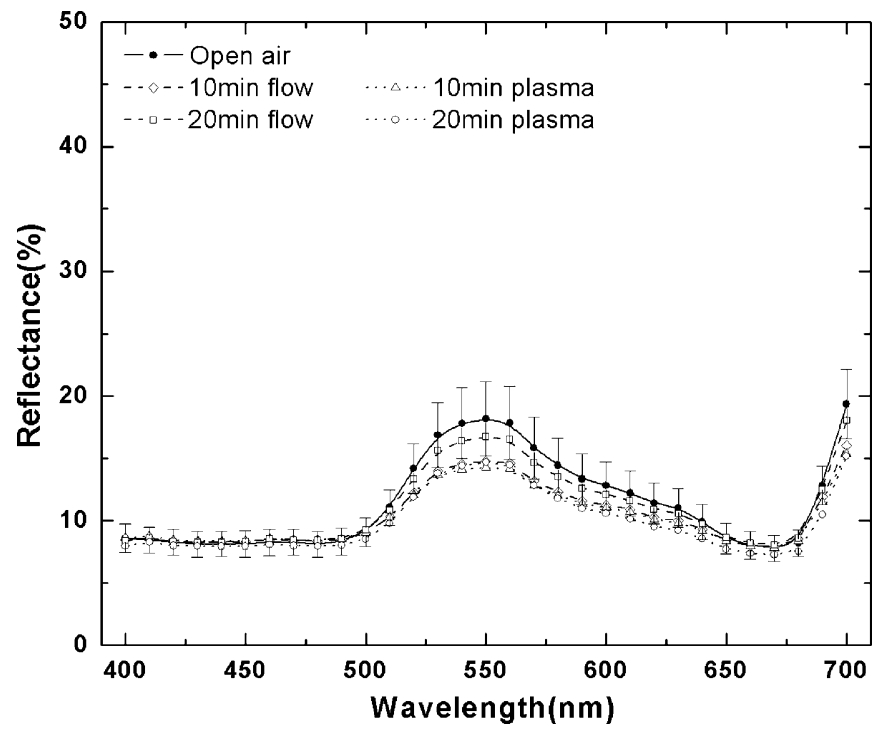

Fig. 6. Reflectance plot from green pepper samples for controls (solid dots), after treatment with unionized gas flows, and after plasma treatment. Variation bars added onto the control curve indicate color variation measured among ten control samples.

bar onto the control curve in Fig. 6. It is clear that the color variation resulting from plasma treatment was smaller than that which would normally be observed by supermarket shoppers. It is conceivable that the inactivation efficacy of APGD could be improved from that in Fig. 3 by use of different plasma treatment conditions. Therefore, discoloration experiments will need to be performed again under these new conditions. It is also necessary to undertake further tests to establish the extent of other plasma-induced surface damages.

\section{CONCLUSION}

In this paper, we have shown that bacteria embedded inside biofilms are still amenable to inactivation by APGD and that plasma inactivation is superior to the use of low-pressure UV sources. We believe that the levels of inactivation can be further improved by altering the gas composition fed to the plasma. Treatment of bell pepper samples with APGD did not have a significant effect on the color of the samples tested. This crucial initial hurdle having been cleared, the way is clear to conduct further, more stringent, quality checks on food samples subjected to APGD treatment.

\section{REFERENCES}

[1] K. G. Donohoe and T. Wydeven, "Plasma polymerization of ethylene in an atmospheric pressure discharge," J. Appl. Polymer Sci., vol. 23, pp. 2591-2601, Sep. 1979.

[2] M. Laroussi, "Sterilization of contaminated matter with an atmospheric pressure plasma," IEEE Trans. Plasma Sci., vol. 24, no. 3, pp. 1188-1191, Jun. 1996.

[3] K. Kelly-Wintenberg, T. C. Montie, C. Brickman, J. R. Roth, A. K. Carr, K. Sorge, L. C. Wadworth, and P. P. Y. Tsai, "Room temperature sterilization of surfaces and fabrics with a one atmosphere uniform glow discharge plasma," J. Ind. Microbiol. Biotechnol., vol. 20, pp. 69-74, Jan. 1998.

[4] H. W. Herrmann, I. Henins, J. Park, and G. S. Selwyn, "Decontamination of chemical and biological warfare (CBW) agents using an atmospheric pressure plasma jet," Phys. Plasmas, vol. 6, no. 5, pp. 2284-2289, May 1999.

[5] P. Koulik, S. Begounov, and S. Goloviatinskii, "Atmospheric plasma sterilization and deodorization of dielectric surfaces," Plasma Chem. Plasma Proc., vol. 19, pp. 311-326, Apr. 1999.

[6] M. Laroussi, I. Alexeff, and W. Kang, "Biological decontamination by nonthermal plasmas," IEEE Trans. Plasma Sci., vol. 28, no. 1, pp. 184-188, Feb. 2000.

[7] J. G. Birmingham and D. J. Hammerstrom, "Bacterial decontamination using ambient pressure nonthermal discharges," IEEE Trans. Plasma Sci., vol. 28, no. 1, pp. 51-55, Feb. 2000.

[8] M. Yamamoto, M. Nishioka, and M. Sadakata, "Sterilization by $\mathrm{H}_{2} \mathrm{O}_{2}$ droplets using corona discharge," J. Electrostat., vol. 55, pp. 158-187, Feb. 2002.

[9] M. Laroussi, J. P. Richardson, and F. C. Dobbs, "Effects of nonequilibrium atmospheric pressure plasmas on the heterotrophic pathways of bacteria and on their cell morphology," Appl. Phys. Lett., vol. 81, no. 4, pp. 772-774, 2002.

[10] E. Stoffels, A. J. Flikweert, W. W. Stoffels, and G. M. W. Kroesen, "Plasma needle: A nondestructive atmospheric plasma source for fine surface treatment of (bio)materials," Plasma Sources Sci. Technol., vol. 11, pp. 383-388, Nov. 2002.

[11] F. J. Trometer, W. J. Neff, O. Franken, M. Heise, M. Neiger, S. H. Liu, G. J. Pietsch, and A. B. Saveljew, "Reduction of bacillus subtilis and Aspergullus Niger spores using nonthermal atmospheric gas discharges," IEEE Trans. Plasma Sci., vol. 30, no. 4, pp. 1416-1423, Aug. 2002.

[12] P. J. Delaquis, S. Stewart, P. M. A. Toivonen, and A. L. Moyls, "Effect of warm, chlorinated water on the microbial flora of shredded iceberg lettuce," Food Res. Int., vol. 32, pp. 7-14, 1999.

[13] J. Du, Y. Han, and R. H. Linton, "Efficacy of chlorine dioxide gas in reducing Escherichia coli O157: H7 on apple surfaces," Food Microbiol., vol. 20, pp. 583-591, 2003.

[14] C. Nguyenthe and F. Carlin, "The microbiology of minimally processed fresh fruits and vegetables," Crit. Rev. Food Sci. Nutrition, vol. 34, pp. 371-401, Apr. 1994.

[15] G. M. Garrity and D. R. Boone, Eds., Bergrey's Manual of Systematic Bacteriology, 2nd ed. Berlin, Germany: Springer, 2001, vol. 1.

Authors photographs and biographies not available at the time of publication. 